\title{
Management of acute type B aortic dissection; ADSORB trial
}

\author{
G. Chad Hughes, MD
}

\begin{abstract}
Objectives: Type B dissection accounts for $25 \%$ to $40 \%$ of all aortic dissections. The current brief review presents an update on this disease, focusing on new data and insights that have come to light in the past 2 years since the topic was last reviewed at the 2012 AATS Aortic Symposium.
\end{abstract}

\begin{abstract}
Methods: A literature search using PubMed (www.ncbi.nih.gov/pubmed) was performed with the search terms "acute type B aortic dissection" and "ADSORB trial," and all English-language articles published in print or available online between 2011 and March 2014 were carefully reviewed. Articles were selected for inclusion on the basis of perceived novel important insights into the pathophysiology and management of acute type B dissection. Data from the ADSORB (Acute Dissection: Stent graft OR Best medical therapy) trial were graciously provided by the ADSORB investigators prior to trial publication.
\end{abstract}

Results: Important new findings regarding acute type B aortic dissection include data on differences between blacks and whites with acute aortic dissection, proposed changes to the current classification system, anatomic predictors of late outcomes, long-term results with thoracic endovascular aortic repair, as well as additional insights into the uncommon but potentially deadly complication of retrograde type A dissection after endovascular repair. Further, early results from the ADSORB trial suggest a benefit for thoracic endovascular repair plus best medical therapy over medical therapy alone for aortic remodeling outcomes 1 year post dissection.

Conclusions: A great deal of important information on acute type B aortic dissection has become available in the past 2 years since the 2012 AATS Aortic Symposium, some of which is summarized in this brief review. Further, it is clear that much additional investigation is needed so we in the aortic disease management community may continue to gain "new insights into an old disease." (J Thorac Cardiovasc Surg 2015;149:S158-62)

This brief review discusses the management of acute type B aortic dissection focusing on new data and insights that have come to light in the past 2 years since the topic was last reviewed at the 2012 AATS Aortic Symposium.

\section{DEMOGRAPHICS OF ACUTE TYPE B AORTIC DISSECTION}

Type B aortic dissection involves the aorta distal to the left subclavian artery and accounts for $25 \%$ to $40 \%$ of all aortic dissections. ${ }^{1}$ However, recent work from the International Registry of Acute Aortic Dissection (IRAD) has demonstrated important differences between blacks and whites with acute aortic dissection. ${ }^{2}$ Specifically, unlike the population as a whole, among black patients, type $\mathrm{B}$ dissection is more common than type A $(52.4 \%$ vs $47.6 \%$, respectively). Consistent with this distribution in dissection subtypes, black patients are more likely to

From the Duke University Medical Center, Durham, NC.

Disclosures: Dr Hughes serves as a consultant and speaker for Medtronic, Inc, Minneapolis, Minn, and W. L. Gore \& Associates, Inc, Flagstaff, Ariz.

Read at The American Association for Thoracic Surgery Aortic Symposium, New York, New York, April 24-25, 2014

Received for publication May 4, 2014; revisions received Aug 27, 2014; accepted for publication Aug 31, 2014; available ahead of print Oct 11, 2014.

Address for reprints: G. Chad Hughes, MD, Center for Aortic Disease, Division of Cardiovascular and Thoracic Surgery, Duke University Medical Center, Box 3051, Durham, NC 27710 (E-mail: gchad.hughes@duke.edu).

$0022-5223 / \$ 36.00$

Copyright (c) 2015 by The American Association for Thoracic Surgery

http://dx.doi.org/10.1016/j.jtcvs.2014.08.083 present with back or abdominal pain than whites. Black patients also more frequently had baseline hypertension and diabetes, as well as a history of cocaine abuse, and more commonly suffered in-hospital acute kidney failure than whites, with an incidence of $36 \%$ versus $20 \%(P<.001)$. Despite these differences, in-hospital and 3-year mortality rate did not differ by race. These findings may have important implications with regards to reducing the incidence of acute dissection among blacks, given their higher incidence of modifiable risk factors. Further, recognition of the fact that blacks are more likely to present with type B rather than type A dissection may lead to earlier imaging in blacks presenting with back or abdominal pain with subsequent earlier diagnosis and potentially improved survival.

\section{CLASSIFICATION SYSTEM}

The Stanford classification system of aortic dissection, described in 1970, has come to be the most widely used in the literature, likely because of its adoption by IRAD in their multiple publications. However, with the advent of thoracic endovascular aortic repair (TEVAR) for the treatment of dissection of the descending thoracic aorta, it has become apparent that the original DeBakey classification, described in 1965, which describes the longitudinal extent of the dissection process in more detail, likely has more utility in this setting. Specifically, DeBakey type IIIa, where the dissection process is confined entirely to the thoracic aorta, should be curable with TEVAR, whereas 


\section{Abbreviations and Acronyms}

ADSORB = Acute Dissection: Stent graft OR Best medical therapy

BMT $=$ best medical treatment

INSTEAD $=$ Investigation of Stent Grafts in Patients With Type B Aortic Dissection

IRAD = International Registry of Acute Aortic Dissection

TEVAR = thoracic endovascular aortic repair

DeBakey IIIb dissections, involving the thoracic and abdominal aorta, will have TEVAR outcomes dependent not only on the thoracic anatomy but also on anatomic features of the abdominal aorta and iliac arteries, including the number of visceral vessels arising from the true lumen as well as the number and size of downstream fenestrations beyond the region treated with TEVAR.

Aortic dissections are also classified by chronicity, based on the time from symptom onset. Historically, acute aortic dissection has been defined as $\leq 14$ days from symptom onset, whereas chronic dissection is considered $>14$ days, derived in part from autopsy data dating back to the 1950s. IRAD recently published ${ }^{3}$ a new temporal classification system of acute dissection whereby a dissection was not considered chronic until $>30$ days after symptom onset, based on an analysis of survival curves demonstrating that survival decreases significantly up to 30 days after presentation. A recent European expert multidisciplinary panel evaluating the treatment of type B aortic dissection ${ }^{4}$ suggested that "acute" should refer to $<2$ weeks, "subacute" from 2 to 6 weeks, and "chronic" $>6$ weeks from symptom onset. Finally, Steuer and colleagues ${ }^{5}$ identified a subgroup of initially uncomplicated acute type B dissection patients who presented with new aortic complications, predominantly rapid aortic enlargement, between 15 and 85 days after symptom onset and suggested adding a subacute, unstable phase, as originally suggested by Borst and colleagues nearly 20 years ago, to the current nomenclature, which they defined as between 15 and 90 days after dissection. Given these recent findings, it is clear that additional work is needed to develop an updated consensus definition of dissection acuity that is based on survival curves and aortic event rates as well as the temporal relationship between the remodeling response of the aorta to endovascular therapy and dissection chronicity.

\section{INTIMOMEDIAL TEARS AND AORTIC DISSECTION PROGNOSIS}

An increasing body of work has emerged over the past several years examining predictors of outcome in acute type B aortic dissection. One factor that has not received much attention until recently is the location of the primary entry tear. Two recent publications ${ }^{6,7}$ have found that for patients with acute type B aortic dissection, those with the primary entry tear located on the concavity (undersurface) of the distal aortic arch more frequently had complicated dissections either at presentation or during initial hospitalization, more commonly had retrograde extension of the dissection process (Figure 1), and more frequently required intervention for management. The authors theorized that the reason for greater retrograde extension in the concavity group is the lack of an anatomic barrier (ie, arch vessels) on the undersurface of the aortic $\operatorname{arch}^{7}$ (Figure 2). Those with a tear on the concavity also had a shorter distance from the tear to the left subclavian artery and a larger false lumen diameter in the mid-descending thoracic aorta. Cox regression analysis demonstrated location of the primary tear on the concavity of the distal arch to be the sole independent predictor of developing a complicated dissection. The authors concluded that localization of the primary tear should be implemented in risk stratification of acute type B aortic dissection and may have an impact on the primary treatment strategy used. They further suggest a modified terminology whereby a primary entry tear on the convexity is referred to as $\mathrm{B} 1$ and a primary tear on the concavity as $\mathrm{B}^{7}$ (Figure 2). Additional work is needed to verify these findings in larger numbers of patients.

The size of the entry tear may also be important with regards to long-term prognosis of an initially uncomplicated type B aortic dissection. Evangelista and colleagues ${ }^{8}$ found that a large $(\geq 10 \mathrm{~mm})$ entry tear in the proximal part of the dissection identified a high-risk subgroup of patients with more rapid aortic expansion and a higher incidence of dissection-related events than those with tears $<10 \mathrm{~mm}$. The reason for this appears to be the higher false lumen pressures achieved in the presence of larger tears. Another interesting finding of this study was that, in a large number of patients, at least 3 years of follow-up was required before dissection complications appeared, suggesting that the hemodynamic or structural factors responsible have a slow effect over time. These findings may also have important implications for future studies intending to compare various treatment strategies for uncomplicated dissection, such as the ADSORB (Acute Dissection: Stent graft OR Best medical therapy) trial discussed below, in that at least 3 years of follow-up will be required to detect any difference between treatment groups. An interesting follow-up study from this group ${ }^{9}$ using in vitro phantoms to study the hemodynamics of the false lumen found that, in the presence of multiple intimomedial tears, flow enters the false lumen through all tears simultaneously during systole, whereas during diastole, flow leaves through all communications, with minimal proximal to distal (or vice versa) flow in the false lumen. In other words, much of the volume entered and left the false lumen from the same tear instead of going from a proximal 


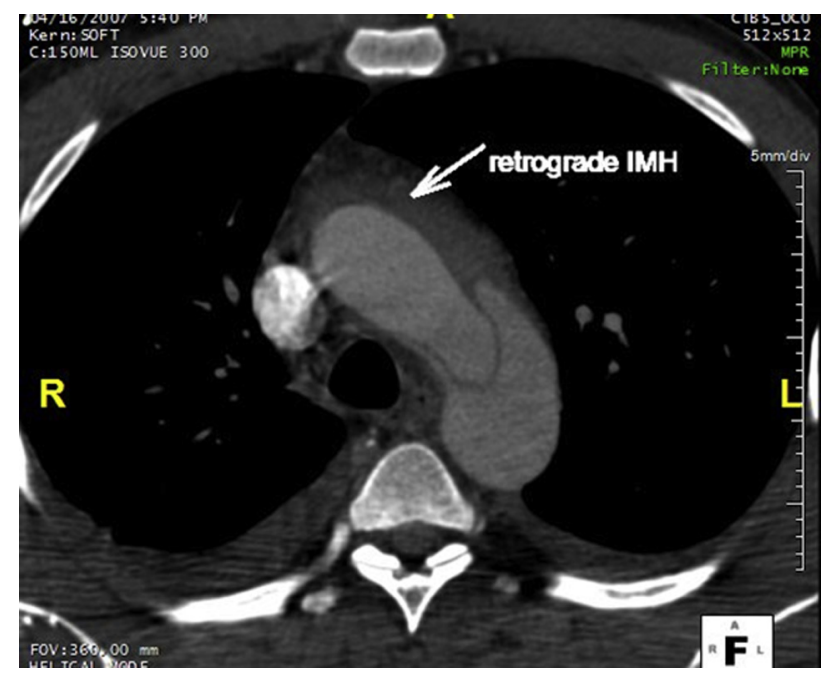

FIGURE 1. Axial contrast-enhanced computed tomographic scan image demonstrating an acute type B dissection with the primary tear on the concavity (undersurface) of the aortic arch. The scan demonstrates evidence for retrogade propagation of an intramural hematoma $(I M H)$ into the more proximal aortic arch, consistent with recently published findings ${ }^{6,7}$ suggesting retrograde propagation of the dissection process to be more common in patients with a primary tear on the concavity of the distal arch.

entry toward a distal exit. As such, false lumen hemodynamics appears to depend heavily on the cumulative tear size as well. These findings would seem discordant with recent work ${ }^{10}$ suggesting that, in acute type B aortic dissection, patients with only 1 entry tear, as compared to those with 2 to 4 tears, have higher rates of aortic growth in follow-up. However, the study did not measure tear size, and it is plausible that those with a single tear had a largediameter entry that was still greater than the cumulative tear size of those with multiple tears, and additional work is needed to reconcile these findings. van Bogerijen and colleagues ${ }^{11}$ have recently published a detailed review of those known predictors of aortic growth in uncomplicated type B dissection, many of which were reviewed at the 2012 AATS Aortic Symposium, ${ }^{1}$ and the reader is referred to this article for further information given the length constraints of the current brief review.

\section{TEVAR}

Multiple consensus statements recommend TEVAR as the treatment of choice for acute complicated type B aortic dissection. ${ }^{12,13}$ Within the past year, the US Food and Drug Administration approved the Gore C-TAG (W. L. Gore \& Associates, Inc, Flagstaff, Ariz) and Medtronic Valiant (Medtronic Vascular, Santa Rosa, Calif) devices for the treatment of acute and chronic, complicated and uncomplicated, dissections of the descending thoracic aorta, although the clinical trials leading to approval of each device included only acute, complicated (rupture or malperfusion) cases.

Our group recently published long-term follow-up data for endovascular repair of acute complicated type B dissection. ${ }^{14}$ Of note, $20 \%$ of patients required 1 or more adjunctive procedures, in addition to TEVAR, to treat malperfusion syndromes, highlighting the increased

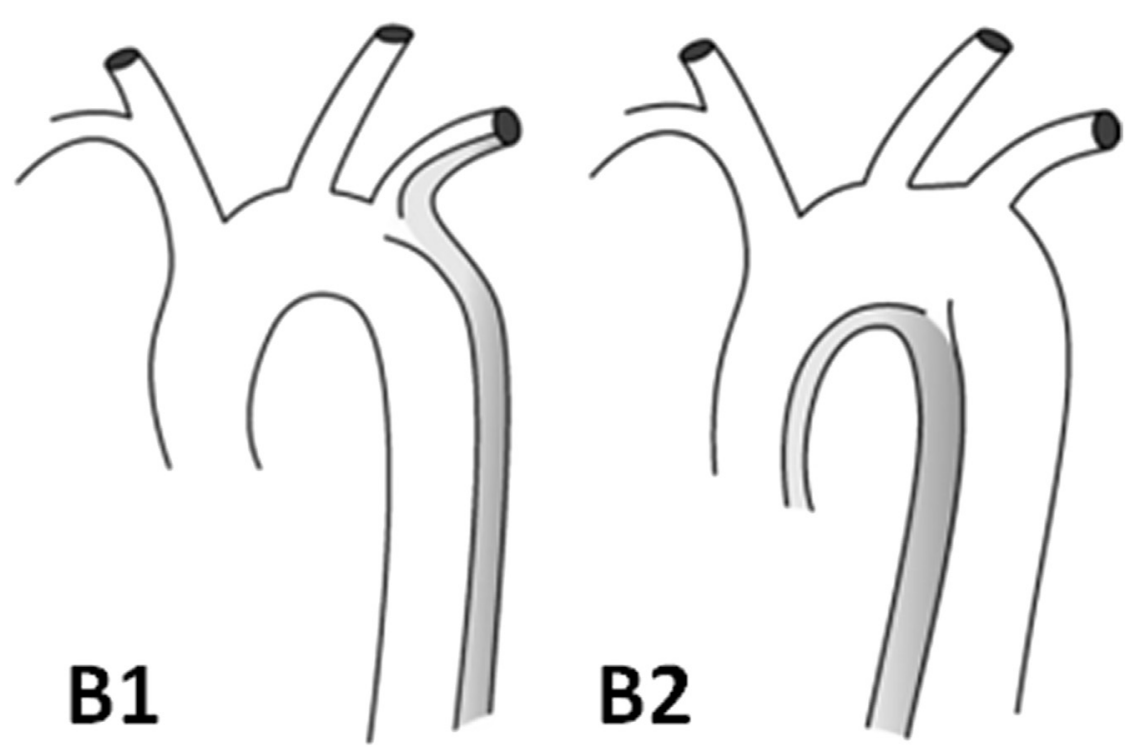

FIGURE 2. Drawing demonstrating acute type B dissection with primary tear on the convexity of the aortic arch (left) and with primary tear on the concavity $(r i g h t)$. Note that for tears on the convexity/outer circumference of the arch, the arch vessels provide an anatomic barrier limiting retrograde extension of the dissection process. In the setting of a primary tear on the concavity/inner circumference of the arch, the lack of anatomic barrier allows retrograde propagation of the dissection process. The authors describing this relationship suggested a modified terminology whereby a primary entry tear on the convexity is referred to as B1 and a primary tear on the concavity as B2. ${ }^{7}$ Figure modified with permission from Loewe and colleagues. 


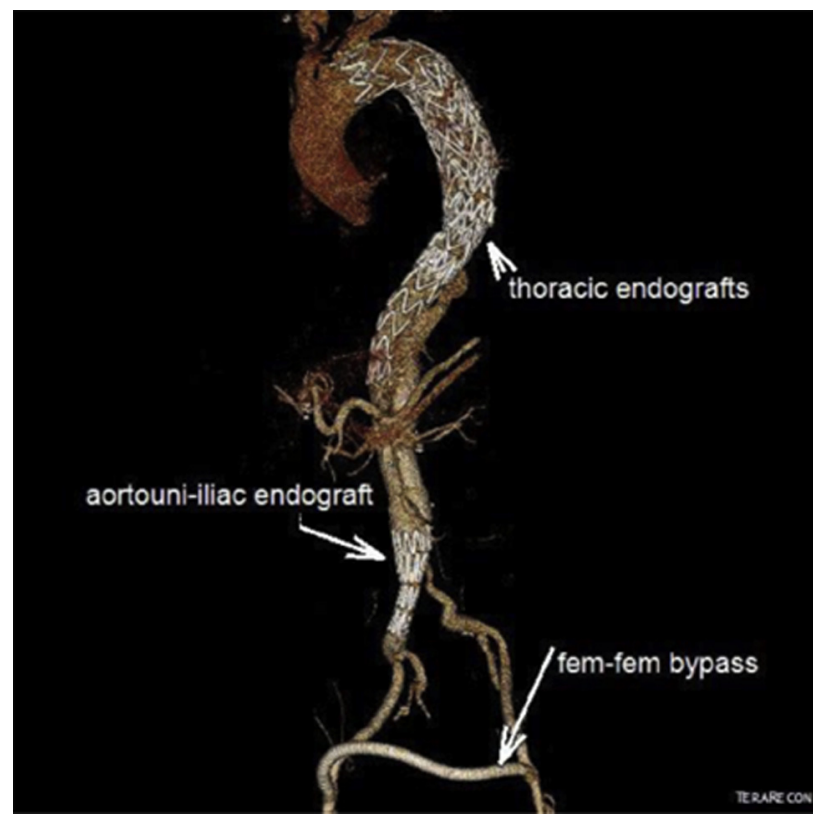

FIGURE 3. Follow-up 3-dimensional reconstruction computed tomographic angiographic image status post endovascular repair of acute complicated type B dissection with iliofemoral malperfusion. The image demonstrates thoracic endografts, an aortouni-iliac endovascular graft, and a femoral-femoral bypass graft, all of which were required to relieve the malperfusion with associated long-segment left iliac occlusion. In the Duke series, ${ }^{14} 20 \%$ of patients required 1 or more adjunctive procedures, in addition to TEVAR, to treat malperfusion syndromes, highlighting the increased complexity of endovascular management for dissection as compared to an aneurysm indication. fem, Femoral.

complexity of endovascular management for dissection as compared to an aneurysm indication (Figure 3). The short-term results were excellent, with no 30-day or inhospital deaths, $2 \%$ stroke and paraplegia or paraparesis, and $4 \%$ new dialysis. Over a median follow-up of 34 months, $26 \%$ of patients required reintervention for various indications, including endoleak, distal fenestrations, and metachronous pathology, although there was no difference in survival between patients who did or did not require reintervention. Aorta-specific survival was $100 \%$ at 7 years, with $84 \%$ overall actuarial survival. These data confirm the excellent short-term outcomes of TEVAR for acute complicated type B dissection and demonstrate the results to be durable over long-term follow-up. However, given that reintervention was required in one quarter of patients, the findings also highlight the importance of lifelong aortic surveillance by an experienced aortic referral center in order to identify and treat complications of the underlying disease process and treatment, as well as new aortic pathologies, as they arise.

\section{ADSORB TRIAL}

As mentioned earlier, TEVAR has now gained broad approval for the treatment of dissection of the descending thoracic aorta, which includes the treatment of uncomplicated dissections, despite a lack of robust data to support this indication. Specifically, no level A evidence exists to support endovascular treatment of acute, uncomplicated DeBakey III dissections, and medical management is still considered the best treatment. ${ }^{1}$ The ADSORB trial is the first randomized trial on acute dissection and compares best medical treatment (BMT) with BMT plus thoracic stent grafting of the primary entry tear in patients with acute uncomplicated type $\mathrm{B}$ dissection. ${ }^{15}$ This multicenter, prospective randomized controlled trial, performed at 17 European centers, randomized 61 patients (31 BMT group, $30 \mathrm{BMT}+$ TEVAR group), aged 18 to 80 years, with uncomplicated (no rupture, malperfusion, or refractory pain) acute type B dissection (penetrating ulcer and intramural hematoma patients excluded), to BMT or BMT plus TEVAR. Important exclusion criteria included retrograde extension of the dissection proximal to the left subclavian artery, anatomy not amenable to TEVAR, severe renal or respiratory insufficiency, and the presence of a connective tissue disorder. The original sample size calculation at trial inception in 2002 called for $250 \mathrm{pa}-$ tients to be randomized, but this was subsequently revised because of slow recruitment as well as newer data from the INSTEAD (Investigation of Stent Grafts in Patients With Type B Aortic Dissection) trial suggesting the study end points could be assessed with a smaller sample size. ${ }^{15}$ The primary study end point is a composite of (1) incomplete or no false lumen thrombosis at 1 year; (2) aortic dilatation ( $\geq 5 \mathrm{~mm}$ or maximum aortic diameter $\geq 55 \mathrm{~mm}$ ) at 1 year; and (3) descending thoracic or abdominal aortic rupture through the 1-year follow-up visit. The alternative hypothesis for the trial is that the proportion of patients being event free at 1 year will be greater for those treated with TEVAR and medical therapy versus medical therapy alone. Analysis of the primary end point is based on intention to treat regardless of treatment received, and morphological analysis of the imaging end points was done by an independent core lab.

The as-yet unpublished results of the trial demonstrated a statistically significant greater freedom from the composite end point at the 1-year study final data lock in the BMT plus TEVAR group as compared to those in the BMT alone cohort $(57 \%$ vs $3 \% ; P<.001)$, although it appears that this was driven almost entirely by a significant difference in false lumen thrombosis between groups, as no significant difference was seen in the aortic dilatation and rupture components of the composite end point. Secondary aortic remodeling end points also favored the BMT plus TEVAR group, with a statistically significant larger true lumen and smaller false lumen, and a trend toward smaller overall aortic diameter, as compared to the BMT alone cohort.

Several criticisms of the ADSORB trial have arisen. First, the definition of false lumen thrombosis was not the same in the BMT plus TEVAR and BMT groups. For patients treated with BMT plus TEVAR, the false lumen was considered 
thrombosed as long as no flow was visualized in the false lumen parallel to the endograft(s), excluding the distal 2 $\mathrm{cm}$, whereas in the BMT group the false lumen was only considered thrombosed if there was no flow in any segment of the thoracic aorta, a difference that would appear to favor the TEVAR group. ${ }^{16}$ In addition, many ${ }^{17}$ feel the surrogate composite end point does not yield clinically useful information on which to base treatment decisions, although it should be acknowledged that ADSORB was conceived as a feasibility study rather than the definitive answer to the question regarding best therapy for acute uncomplicated type B dissection. Finally, given the small sample size and short duration of follow-up, the trial is not powered to detect differences in aortic-related and all-cause mortality, a study that would require $>2000$ subjects and several years of follow-up.

\section{RETROGRADE TYPE A DISSECTION}

Retrograde type A dissection remains an uncommon but potentially deadly complication of TEVAR and is one of the prime factors limiting the routine use of this therapy for uncomplicated type B dissection. Prior work from our group ${ }^{18}$ has identified a dissection indication for TEVAR to be one of the key risk factors for developing this complication, especially in the setting of an even mildly dilated $(\geq 4.0 \mathrm{~cm})$ ascending aorta. It is also important to note that prior data from IRAD has demonstrated that $40 \%$ of patients with acute type B dissection have an ascending aortic diameter $>4 \mathrm{~cm},{ }^{19}$ thus placing them at additional risk. Another potentially important risk factor applicable to the use of TEVAR for dissection, as mentioned above, is that patients with the primary entry tear on the concavity of the aortic arch are more likely to develop retrograde propagation of the dissection, ${ }^{6,7}$ and a recent series by Gorlitzer and colleagues ${ }^{20}$ suggests that this risk also applies to TEVAR in the setting of a concavity tear. This finding, if confirmed in larger studies, will have significant implications with regards to the use of TEVAR for type B dissection in the future.

In summary, a great deal of important information has come to light in the past 2 years, since the 2012 AATS Aortic Symposium, ${ }^{1}$ some of which has been summarized in this brief review. Further, it is clear that much additional investigation is needed to continue to gain "new insights into an old disease," as the original paper from IRAD ${ }^{21}$ eloquently described in 2000 .

\section{References}

1. Hughes GC, Andersen ND, McCann RL. Management of acute type B aortic dissection. J Thorac Cardiovasc Surg. 2013;145(Suppl):S202-7.
2. Bossone E, Pyeritz RE, O'Gara P, Harris KM, Braverman AC, Pape L, et al. Acute aortic dissection in blacks: Insights from the international registry of acute aortic dissection. Am J Med. 2013;126:909-15.

3. Booher AM, Isselbacher EM, Nienaber CA, Trimarchi S, Evangelista A, Montgomery DG, et al. The IRAD classification system for characterizing survival after aortic dissection. Am J Med. 2013;126:730.e19-24.

4. Fattori R, Cao P, DeRango P, Czerny M, Evangelista A, Nienaber C, et al. Interdisciplinary expert consensus document on management of type B aortic dissection. J Am Coll Cardiol. 2013;61:1661-78.

5. Steuer J, Björck M, Mayer D, Wanhainen A, Pfammatter T, Lachat M. Distinction between acute and chronic type B aortic dissection: Is there a sub-acute phase? Eur J Vasc Endovasc Surg. 2013;45:627-31.

6. Weiss G, Wolner I, Folkmann S, Sodeck G, Schmidli J, Grabenwöger M, et al. The location of the primary entry tear in acute type B aortic dissection affects early outcome. Eur J Cardiothorac Surg. 2012;42:571-6.

7. Loewe C, Czerny M, Sodeck GH, Ta J, Schoder M, Funovics M, et al. A new mechanism by which an acute type B aortic dissection is primarily complicated, becomes uncomplicated, or remains uncomplicated. Ann Thorac Surg. 2012;93: 1215-22.

8. Evangelista A, Salas A, Ribera A, Ferreira-González I, Cuellar H, Pineda V, et al. Long-term outcome of aortic dissection with patent false lumen: Predictive role of entry tear size and location. Circulation. 2012;125:3133-41.

9. Rudenick PA, Bijnens BH, García-Dorado D, Evangelista A. An in vitro phantom study on the influence of tear size and configuration on the hemodynamics of the lumina in chronic type B aortic dissections. J Vasc Surg. 2013;57:464-74.

10. Tolenaar JL, van Keulen JW, Trimarchi S, Jonker FHW, van Herwaarden JA, Verhagen HJM, et al. Number of entry tears is associated with aortic growth in type B dissections. Ann Thorac Surg. 2013;96:39-42.

11. van Bogerijen GHW, Tolenaar JL, Rampoldi V, Moll FL, van Herwaarden JA, Jonker FHW, et al. Predictors of aortic growth in uncomplicated type B aortic dissection. J Vasc Surg. 2014;59:1134-43.

12. Coady MA, Ikonomidis JS, Cheung AT, Matsumoto AH, Dake MD, Chaikof EL, et al. Surgical management of descending thoracic aortic disease: open and endovascular approaches: a scientific statement from the American Heart Association. Circulation. 2010;121:2780-804.

13. Svensson LG, Kouchoukos NT, Miller DC, Bavaria JE, Coselli JS, Curi MA, et al. Expert consensus document on the treatment of descending thoracic aortic disease using endovascular stent-grafts. Ann Thorac Surg. 2008;85:S1-41.

14. Hanna JM, Andersen ND, Ganapathi AM, McCann RL, Hughes GC. Five-year results for endovascular repair of acute complicated type B aortic dissection. $J$ Vasc Surg. 2014;59:96-106.

15. Brunkwall J, Lammer J, Verhoeven E, Taylor P. ADSORB: A study on the efficacy of endovascular grafting in uncomplicated acute dissection of the descending aorta. Eur J Vasc Endovasc Surg. 2012;44:31-6.

16. Voûte MT, Gonçalves FB, Verhagen HJM. Commentary on "ADSORB: A study on the efficacy of endovascular grafting in uncomplicated acute dissection of the descending aorta." Eur J Vasc Endovasc Surg. 2012;44:37.

17. Hinchliffe RJ, Thompson MM. ADSORB: A prospective randomised study on the efficacy of endovascular grafting vs. best medical treatment in uncomplicated acute dissection of the descending aorta. Eur J Vasc Endovasc Surg. 2012;44: 38-9.

18. Williams JB, Andersen ND, Bhattacharya SD, Scheer E, Piccini JP, McCann RL, et al. Retrograde ascending aortic dissection as an early complication of thoracic endovascular aortic repair. J Vasc Surg. 2012;55:1255-62.

19. Booher AM, Isselbacher EM, Nienaber CA, Froehlich JB, Trimarchi S, Cooper JV, et al. Ascending thoracic aorta dimension and outcomes in acute type B dissection (from the International Registry of Acute Aortic Dissection [IRAD]). Am J Cardiol. 2011;107:315-20.

20. Gorlitzer M, Weiss G, Moidl R, Folkmann S, Waldenberger F, Czerny M, et al. Repair of stent graft-induced retrograde type A aortic dissection using the Evita open prosthesis. Eur J Cardio-Thorac Surg. 2012;42:566-70.

21. Hagan PG, Nienaber CA, Isselbacher EM, Bruckman D, Karavite DJ, Russman PL, et al. The International Registry of Acute Aortic Dissection (IRAD): new insights into an old disease. JAMA. 2000;283:897-903. 